\title{
Valoración de la capacidad aeróbica de estudiantes universitarios*
}

\author{
Julio Alejandro Gómez-Figueroa* \\ Alejandro Barradas-Peregrina \\ Santiago Castineyra-Mendoza* \\ Luis Quintana-Rivera \\ José Moncada-Jiménez
}

Recibido: junio 25 del 2020 • Aceptado: julio 27 del 2020

\begin{abstract}
Artículo de investigación, sin financiamiento. La investigación se inició en agosto de 2018 y finalizó en julio de 2019.

Citar como: Gómez-Figueroa, J., Barradas-Peregrina, A., Castineyra-Mendoza, S., Quintana-Rivera, L. y Moncada-Jiménez, J. (2020). Valoración de la capacidad aeróbica de estudiantes universitarios. Revista de Investigación Cuerpo, Cultura y Movimiento, 10(2), 79-95. DOI: https://doi.org/10.15332/2422474x/6227
\end{abstract}

"* Doctor en Educación; director de la de la Facultad de Educación Física, Deporte y Recreación de la Universidad Veracruzana, México. Líder del Cuerpo Académico UV-CA- 292. Línea de Investigación Actividad Física para la Salud. Correo electrónico: julgomez@uv.mx, ORCID: 0000-0001-8400-6087

*** Máster en Ciencias Aplicadas a la Actividad Física. Docente de tiempo completo Titular B, de la Facultad de Educación Física, Deporte y Recreación de la Universidad Veracruzana, México. Correo electrónico: albarradas@uv.mx, oRCID: 0000-0002-8651-594X

"***** Máster en Ciencias Aplicadas al Deporte. Técnico académico tiempo completo Titular B de la Facultad de Educación Física, Deporte y Recreación de la Universidad Veracruzana, México. Colaborador del Cuerpo Académico UV-CA-292. Correo electrónico: scastineyra@uv.mx, ORCID: 0000-0002-8651-594X

*:*:**: Doctor en Educación; secretario académico de la de la Facultad de Educación Física, Deporte y Recreación de la Universidad Veracruzana, México. Colaborador del Cuerpo Académico UV-CA- 292. Correo electrónico: lquintana@uv.mx, orCID: 0000-0003-3831-5932

${ }^{* * * * * * * *}$ Doctor en Ciencias Biomédicas; investigador del Centro de Investigaciones de Ciencias del Movimiento Humano, Universidad de Costa Rica. Docente de la Escuela de Educación Física y Deportes de la Universidad de Costa Rica. Correo electrónico: jose.moncada@ucr.ac.cr, ORCID: 0000-0001-9807-5163 


\section{Resumen}

Algunas universidades en el mundo realizan pruebas de aptitud física a sus estudiantes de primer ingreso. Una de esas pruebas permite determinar la capacidad aeróbica, un componente de la aptitud física relacionado con la salud y la reducción de riesgos cardiovasculares. El propósito del estudio fue determinar la capacidad aeróbica de estudiantes de primer ingreso de la Universidad Veracruzana en México. Participaron 1004 estudiantes de ambos sexos, quienes completaron la prueba aeróbica de Cooper. Se encontró que el $63.0 \%$ de los participantes se ubicó en niveles bajos; $25.6 \%$, en niveles medios, y $11.4 \%$, en un nivel bueno. Se concluye que los estudiantes de primer ingreso poseen una baja capacidad aeróbica, lo que impactará en su desempeño físico si participan en actividades deportivas. Además, esta inadecuada condición se manifiesta en hombres y mujeres, por lo que es necesario implementar estrategias de ejercicio físico para que impacte en la prevención y cuidado de la salud física, coadyuvando en la disminución de los riesgos de enfermar y colaborando en la reducción del gasto en servicios de salud de la comunidad estudiantil universitaria.

Palabras claves: actividad física, consumo máximo de oxígeno, estudiantes. 


\title{
Assessment of the Aerobic Capacity of University Students
}

\begin{abstract}
Some universities in the world conduct physical fitness tests to students during their freshmen year. One of these tests determines aerobic capacity, a component of physical fitness related to health and cardiovascular risk reduction. The purpose of the study was to determine the aerobic capacity of freshmen students at the University of Veracruz, in Mexico. Participants were 1004 students of both genders, who completed the Cooper's test. It was found that $63.0 \%$ of the participants were at low levels of aerobic capacity; $25.6 \%$, at medium levels; and $11.4 \%$, at good levels. In conclusion, freshmen students showed low aerobic capacity, which will impact their physical performance if they participate in sports activities. In addition, this inadequate condition is found in men and women; thus, it is necessary to implement physical exercise strategies that impact prevention and physical health care, helping to reduce the risk of illness and the money spent on health services by the university student community.
\end{abstract}

Keywords: physical activity, maximum oxygen consumption, students. 


\section{Introducción}

Existe contundente evidencia científica acerca de las consecuencias negativas que trae para la salud el tener un estilo de vida con hábitos inapropiados (Carrasco-Alarcón, Martínez-Salazar, Caniuqueo-Vargas y Díaz-Bustos, 2014; Durán, Valdés, Godoy y Herrera, 2014; García-Laguna, García-Salamanca, Tapiero-Paipa y Ramos, 2012; García-Soidán y AlonsoFernández, 2011; George, Fisher y Vehrs, 2005; Melo-Mccormick y Rueda-Ochoa, 2007). Se sabe que estos estilos afectan la calidad de vida, el estado de salud y la aptitud física general. También se reconoce que, en la actualidad, la población de personas jóvenes posee estilos de vida negativos que impactan en su salud, como, por ejemplo, un elevado nivel de sedentarismo, inadecuados hábitos de alimentación, tabaquismo e inadecuado manejo del estrés (Carpio-Rivera, Solera-Herrera, Salicetti-Fonseca, Hernández-Elizondo y Moncada-Jiménez, 2016; Fernández-Ramírez y Moncada-Jiménez, 2003).

Los estilos de vida sedentarios son considerados patrones de conducta influenciados por factores socioculturales que no promueven la realización de actividad física, lo que desencadena una amplia gama de problemas de salud (García-Laguna et al., 2012). Existe evidencia contundente que indica una estrecha relación entre numerosas patologías derivadas de un estilo de vida sedentario; por ejemplo, el sedentarismo se asocia con aumentos en la adiposidad (i. e., sobrepeso y obesidad), síndrome metabólico, algunos tipos de cáncer, fragilidad ósea, baja cognición en la edad adulta mayor y baja fuerza muscular en estudiantes universitarios (Carrasco-Alarcón et al., 2014; Chastin, Mandrichenko, Helbostadt y Skelton, 2014; Hartman, Karssemeijer, van Diepen, Olde Rikkert y Thijssen, 2018; Kerr, Anderson y Lippman, 2017; Lemes et al., 2019; Silfee, Lemon, Lora y Rosal, 2017).

Se ha reconocido que la actividad física es una conducta asociada a la buena salud; de hecho, se le considera como una polipíldora, lo que significa que se asocia a numerosos beneficios para la salud a un bajo costo (Fiuza-Luces, Garatachea, Berger y Lucia, 2013; Pareja-Galeano, Garatachea y Lucia, 2015; Sanchis-Gomar, Fiuza-Luces y Lucia, 2015). Por lo tanto, conocer el estado de salud física de los individuos proporciona información valiosa acerca de la capacidad de las personas para ejecutar actividades físicas (Riebe et al., 2015), y uno de esos elementos es la capacidad aeróbica. 
La capacidad aeróbica, medida por medio del consumo máximo de oxígeno $\left(\mathrm{VO}_{2} \mathrm{máx}\right)$, se refiere a la capacidad del sistema cardiovascular para transportar y utilizar el oxígeno para realizar el trabajo muscular (American College of Sports Medicine [ACSM], 2018). Se ha establecido epidemiológicamente que una persona saludable debería tener una capacidad aeróbica que le permita responder adecuadamente a las exigencias de la vida diaria sin fatigarse excesivamente (Melo-Mccormick y Rueda-Ochoa, 2007). De esta forma, un mayor $\mathrm{VO}_{2}$ máx se asocia con una mayor capacidad para realizar ejercicio aeróbico como, por ejemplo, en ciclistas, corredores de fondo y nadadores. En estudiantes universitarios rumanos y serbios, se ha encontrado una alta correlación entre el $\mathrm{VO}_{2}$ máx y el índice de aptitud física (Dana, Goran, Dusan y Adela, 2015).

La medición de la capacidad aeróbica se realiza por medio de un gold standard en un laboratorio, con equipo costoso (i. e., carro metabólico, banda sin fin, cicloergómetro) y personal altamente especializado (i.e., fisiólogo del ejercicio, médico). Aunque esta prueba de laboratorio provee la valoración más directa de la capacidad aeróbica, se han diseñado pruebas de campo como alternativas para reducir los costos, el tiempo de medición y evaluación, especialmente en grupos de personas (ACSM, 2018; GómezFigueroa, Anaya-Méndez, Parrazal-Cobos y Rivera-Girón, 2015). Una de estas pruebas es la denominada prueba aeróbica de Cooper (Cooper, 1968), desarrollada inicialmente para el ámbito militar, pero que después fue ganando popularidad en el ámbito educativo y deportivo (Bandyopadhyay, 2015). Esta prueba presenta valores adecuados de validez y confiabilidad al compararlo con el gold standard (Mayorga-Vega, Bocanegra-Parrilla, Ornelas y Viciana, 2016). En este contexto, el propósito del estudio fue evaluar la capacidad aeróbica en jóvenes universitarios de primer ingreso de la Universidad Veracruzana en México.

\section{Metodología}

El estudio fue exploratorio, descriptivo y transversal (Campbell y Stanley, 1963), con el que se midió la variable de capacidad aeróbica sin que existiera una intervención por parte de los investigadores. 


\section{Participantes}

En el estudio participaron 1004 universitarios de nuevo ingreso de la Universidad Veracruzana de la Región Veracruz-Boca del Río. Los participantes tenían edades entre 17 y 38 años, de los cuales se contó con 509 hombres $(50.7 \%)$ y 495 mujeres $(49.3 \%)$. Esta muestra representó el $36.7 \%$ de la matrícula total del 2018 . El $68.0 \%$ provenían de ciudades diferentes al puerto de Veracruz y el $32.0 \%$ es originario del mismo.

\section{Instrumento y procedimientos de medición}

La prueba de capacidad aeróbica utilizada fue el test de Cooper, que consiste en recorrer la mayor distancia posible sobre una superficie plana, libre de obstáculos, caminando o corriendo durante 12 min (Cooper, 1968). Diversos investigadores (Alvero-Cruz, Giráldez García y Carnero, 2017; Das, 2013; Gamboa-Carmona y Mendoza-Romero, 2009; Mayorga-Vega et al., 2016) sugieren utilizar la prueba de Cooper en grupos numerosos, ya que se adapta fácilmente y requiere equipamiento mínimo; además de brindar valores del $\mathrm{VO}_{2}$ máx válidos $(\mathrm{r}=0.90)$ y confiables $\left(\mathrm{r}_{\text {test, re-test }}=0.94\right)$ (Miller, 1996).

El protocolo seguido en este estudio respetó los derechos de los participantes siguiendo normas internacionales para la protección de sujetos que participan en investigaciones (World Medical Association, 2014). De esta forma, los participantes completaron y firmaron un consentimiento informado para ser parte del estudio. La recopilación de la información se realizó entre agosto y diciembre del 2018 , con la participación de los programas educativos del área técnica, ciencias de la salud, económico administrativo, agropecuaria y humanidades, siendo estas las Facultades de Ingeniería, Ciencias y Técnicas de la Comunicación, Pedagogía, Veterinaria y Zootecnia, Odontología, Educación Física, Deporte y Recreación, Enfermería, Nutrición, Medicina, Psicología, Contaduría y Administración.

La administración de las pruebas se coordinó realizando un cronograma de citas junto con el director del Centro de Servicios en Salud, que posteriormente se difundió entre los coordinadores de cada facultad de la región Veracruz-Boca del Río. A los participantes se les solicitó abstenerse de realizar actividad física vigorosa e intensa 24 h previas a su cita, así como de no realizar una ingesta de alimentos sólidos tres horas antes de la ejecución, 
y mantener una ingesta de líquidos rehidratantes suficientes durante el día. Así, los estudiantes fueron citados en la Facultad de Educación Física, Deporte y Recreación, en donde completaron el formulario de consentimiento informado y se recopiló información demográfica (i. e., edad, sexo), y la unidad académica a la que pertenecían.

Posteriormente, realizaron un calentamiento general y específico guiado por profesionales de educación física antes de realizar la prueba de Cooper. Esta prueba se llevó a cabo en condiciones ambientales entre $23{ }^{\circ} \mathrm{C}$ y $29^{\circ} \mathrm{C}$, y con una humedad relativa de entre $40 \%$ y $70 \%$. Los participantes fueron ubicados en una pista atlética de $400 \mathrm{~m}$, se les dio la señal de inicio y, al final de los 12 min de duración de la prueba, se midió la distancia total recorrida en metros. Esa distancia permitió realizar una valoración cualitativa de la capacidad aeróbica por sexo y edad de los participantes (tabla 1).

Tabla 1. Distancias de referencia para la valoración de la prueba de Cooper por sexo y edad

\begin{tabular}{ccccc}
\hline \multirow{3}{*}{ Clasificación } & & \multicolumn{3}{c}{ Grupo de edad (años) } \\
\cline { 3 - 5 } Muy Bajo & Sexo & $\mathbf{1 3 - 1 9}$ & $\mathbf{2 0 - 2 9}$ & $30-39$ \\
& Masculino & $<2100$ & $<1950$ & $<1900$ \\
& Femenino & $<1600$ & $<1550$ & $<1500$ \\
Bajo & Masculino & $2100-2200$ & $1950-2100$ & $1900-2100$ \\
& Femenino & $1600-1900$ & $1550-1800$ & $1500-1700$ \\
Medio & Masculino & $2200-2500$ & $2100-2400$ & $2100-2350$ \\
& Femenino & $1900-2100$ & $1800-1950$ & $1700-1900$ \\
\multirow{3}{*}{ Bueno } & Masculino & $2500-2750$ & $2400-2650$ & $2350-2500$ \\
& Femenino & 21 y0-2300 & $1950-2150$ & $1900-2100$ \\
\multirow{2}{*}{ Excelente } & Masculino & $2750-3000$ & $2650-2850$ & $2500-2650$ \\
& Femenino & $2300-2450$ & $2150-2350$ & $2100-2250$ \\
& Masculino & $>3000$ & $>2850$ & $>2700$ \\
& Femenino & $>2450$ & $>2350$ & $>2250$ \\
\hline
\end{tabular}

Nota: Los valores son distancias en metros para personas no entrenadas (Manso, Valdivielso y Caballero, 1996).

Fuente: elaboración propia. 


\section{Análisis estadísticos}

Los análisis estadísticos se realizaron utilizando el programa SPSS versión 21 (IBM Corp., Armonk, Nueva York, USA). Las estadísticas descriptivas se presentan como frecuencias y porcentajes para las variables categóricas y como la media y desviación estándar para la edad de los participantes. Se realizaron pruebas no paramétricas de $\mathrm{Chi}^{2}$ para estudiar las proporciones de respuestas entre las variables nominales.

\section{Resultados}

La edad promedio de los participantes fue de $18.6 \pm 1.2$ años (mínimo $=17$, máximo $=22$ ). Los participantes provenían de 11 planes de estudio pertenecientes a cinco áreas del conocimiento: a) Ciencias de la Salud, b) Económico Administrativo, c) Agropecuaria, d) Técnica, y e) Humanidades. En la tabla 2 se presenta la distribución de los participantes de acuerdo con el plan de estudio cursado.

Tabla 2. Distribución de los participantes por tipo plan de estudio

\begin{tabular}{ccc}
\hline Licenciatura & Frecuencia & \% \\
\hline Administración & 218 & 21.7 \\
Medicina & 140 & 13.9 \\
Ingeniería & 147 & 14.6 \\
Veterinaria & 91 & 9.0 \\
Contaduría & 78 & 7.8 \\
Pedagogía & 74 & 7.7 \\
Odontología & 73 & 7.2 \\
Educación Física & 72 & 7.1 \\
Comunicaciones & 65 & 6.4 \\
Enfermería & 35 & 3.4 \\
Nutrición & 11 & 1.19 \\
Total & 1004 & 100 \\
\hline
\end{tabular}

Fuente: elaboración propia. 
Los participantes fueron categorizados por grupo etario con la finalidad de tener un análisis objetivo, siendo el mayor porcentaje el de $57.3 \%$ de los sujetos entre 17 y 18 años ( $\mathrm{n}=575), 31.7 \%$ en el rango de 19 a 20 años $(\mathrm{n}=318)$ y el $11.1 \%$ mayores de 21 años $(n=111)$.

En la tabla 3 se muestra la categorización de la capacidad aeróbica por sexo. El $31 \%$ de los hombres tuvo un nivel muy bajo; el $25.7 \%$, un nivel bajo; el $28.1 \%$, un nivel medio, y el $14.1 \%$ mostró un nivel bueno. Por otra parte, el $38.3 \%$ de las mujeres tuvo valores muy bajos; el $30.1 \%$, un nivel bajo; el $23 \%$, un nivel medio; y el $8.48 \%$, un nivel bueno.

Tabla 3. Distribución y categorización de la capacidad aeróbica de los participantes por sexo

\begin{tabular}{cccccc}
\cline { 2 - 4 } & \multicolumn{4}{c}{ Capacidad aeróbica } & \multirow{2}{*}{ Total } \\
\cline { 2 - 5 } & Muy bajo & Bajo & Medio & Bueno & \\
\hline Masculino (n) & 159 & 135 & 143 & 72 & 509 \\
Femenino (n) & 190 & 149 & 114 & 42 & 495 \\
Totales (n) & 349 & 284 & 257 & 114 & 1004 \\
\hline
\end{tabular}

Fuente: elaboración propia.

El análisis de $\mathrm{Chi}^{2}$ indicó que existen proporciones distintas en las valoraciones de la capacidad aeróbica entre los estudiantes de los diferentes planes de estu$\operatorname{dio}\left(\chi^{2}=278.49, p \leq 0.001\right)$. En los planes educativos de Contaduría, Medicina, Nutrición, Enfermería, Odontología, Veterinaria, Ciencias y Técnicas de la Comunicación, las proporciones de estudiantes son similares en todas las categorías de clasificación de la capacidad aeróbica.

En el plan de estudios de Pedagogía, se encontró una mayor proporción de estudiantes en las categorías de clasificación de la capacidad aeróbica bajo y regular, comparado con las categorías bueno, muy bueno y excelente. En el plan de estudios de Administración, se encontró una mayor proporción de estudiantes en la categoría de clasificación de la capacidad aeróbica bajo, comparado con las categorías de regular, bueno, muy bueno y excelente. En el plan de estudios de Ingeniería, se encontró una mayor proporción de estudiantes en las categorías de clasificación de la capacidad aeróbica regular, bueno y muy bueno, comparado con las categorías bajo y excelente. 
En el plan de estudios de Educación Física, se encontró una mayor proporción de estudiantes en las categorías de clasificación de la capacidad aeróbica excelente comparado con las categorías bajo, regular, bueno y muy bueno. La categoría muy bueno también tuvo una mayor proporción de estudiantes que las categorías bajo, regular y bueno. No hubo diferencias en las proporciones de estudiantes entre las categorías bajo, regular y bueno.

$\mathrm{El}$ análisis de $\mathrm{Chi}^{2}$ indicó que existen proporciones distintas en las valoraciones de la capacidad aeróbica en hombres y en mujeres $\left(\chi^{2}=263.51\right.$, $p \leq 0.001)$. Hay diferencias en las proporciones de hombres entre las categorías bajo, regular, bueno y muy bueno. La proporción de hombres en la categoría excelente es menor que en las categorías bajo y regular. Lo mismo se encuentra en las mujeres (Figura 1).

Figura 1. Proporción de estudiantes masculinos y femeninos de acuerdo con la clasificación de la capacidad aeróbica

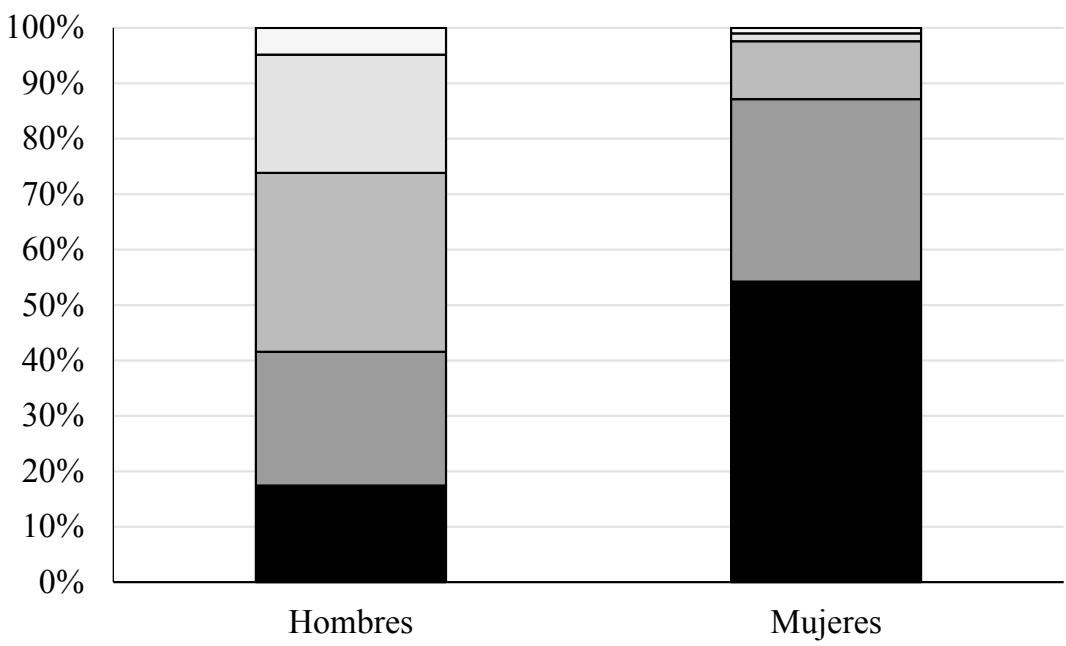

- Deficiente $\square$ Regular $\square$ Bien $\square$ Muybien $\square$ Excelente

Fuente: elaboración propia. 


\section{Discusión}

El objetivo del presente estudio fue evaluar la capacidad aeróbica en jóvenes universitarios de primer ingreso de la Universidad Veracruzana, en México. Para determinar esta cualidad de la aptitud física, se utilizó la prueba de 12 min de Cooper. El hallazgo principal fue que más del $85 \%$ de las mujeres presentó una capacidad aeróbica catalogada entre deficiente y regular. Por su parte, $\sim 40 \%$ de los hombres también tuvieron esa clasificación.

Diversos estudios evidencian la utilidad del test de Cooper como una prueba indirecta para evaluar la capacidad aeróbica (Bandyopadhyay, 2015). Por ejemplo, en la India, 30 mujeres con una edad promedio de $18 \pm 1.5$ años realizaron la prueba de Cooper y se encontraron valores promedio de $41.3 \pm 4.4 \mathrm{~mL} \cdot \mathrm{kg}^{-1} \cdot \mathrm{min}^{-1}{ }^{1}$ (zona urbana) y $50.2 \pm 5.8 \mathrm{~mL} \cdot \mathrm{kg}^{-1} \cdot \mathrm{min}^{-1}{ }^{1}$ (zona rural) (Das, 2013). Para las mujeres de zona urbana, estos valores se ubican en el percentil 70 (bueno) de las normas del ACSM (2018) y el percentil 75 de la Clínica Cooper (Kaminsky, Arena y Myers, 2015). Por su parte, para las mujeres de zona rural, los valores de la capacidad aeróbica se ubican en el percentil 95 (superior) de las normas del ACSM (2018) y sobre el percentil 95 de la Clínica Cooper (Kaminsky et al., 2015).

En Brasil, Koury et al. (2016) evaluaron a jóvenes militares y encontraron que el $64 \%$ de los cadetes del ejército se clasificaron con excelente capacidad aeróbica $\left(\mathrm{VO}_{2}\right.$ máx $\left.=53.9 \pm 3.0 \mathrm{~mL} \cdot \mathrm{kg}^{-1} \cdot \mathrm{min}^{-1}\right)$ medido por medio de la prueba de Cooper, y categorizado en el percentil 85 (excelente) por el ACSM (2018) y entre el percentil 75 y 90 por la Clínica Cooper (Kaminsky et al., 2015). Valores similares de capacidad aeróbica han sido reportados en estudiantes universitarios masculinos costarricenses $(\mathrm{n}=138)$ (Carpio-Rivera et al., 2016).

En otro estudio en jóvenes universitarios de Brasil (Michell, Samaria, Junior Rudy, Danyela y Dantas, 2014), se utilizó la prueba de Cooper en 71 hombres no atléticos, quienes obtuvieron un promedio de $\mathrm{VO}_{2}$ máx $=34.5 \pm 0.8$ $\mathrm{mL} \cdot \mathrm{kg}^{-1} \cdot \mathrm{min}^{-1}$, valor catalogado en el percentil 20 (pobre) por el ACSM (2018) y cercano al percentil 25 de las normas de la Clínica Cooper (Kaminsky et al., 2015). Para obtener esa predicción, se utilizó la fórmula: $\mathrm{VO}_{2}$ máx $(\mathrm{ml} \cdot \mathrm{kg}$ $\left.{ }^{1} \cdot \min ^{-1}\right)=($ distancia recorrida en metros -504.1$) / 44.9$.

En un grupo de 58 hombres sedentarios pertenecientes a una universidad en la India, se encontró un promedio de VO2máx de $42.8 \pm 4.0 \mathrm{~mL} \cdot \mathrm{kg}^{-1} \cdot \mathrm{min}^{-1}$, valor catalogado en el percentil 40 (bajo) por el ACSM (2018) y cercano al 
percentil 50 de las normas de la Clínica Cooper (Kaminsky et al., 2015). Esos estudiantes siguieron el mismo protocolo de Cooper utilizado en el presente estudio y, para predecir el $\mathrm{VO}_{2}$ máx, utilizaron la fórmula descrita por Cooper (1968): $\mathrm{VO}_{2} \operatorname{máx}\left(\mathrm{ml} \cdot \mathrm{kg}^{-1} \cdot \mathrm{min}^{-1}\right)=(22.351 \times$ distancia recorrida en $\mathrm{km})$ - 11.288 .

En el presente estudio no se utilizaron fórmulas de predicción del $\mathrm{VO}_{2}$ máx calculadas a partir de la distancia recorrida en la prueba de Cooper, ya que se ha encontrado que las fórmulas de predicción de variables de la aptitud física no son extrapolables entre las poblaciones, por lo que se prefiere utilizar fórmulas desarrolladas específicamente para la población de interés (CarpioRivera, Hernández-Elizondo, Salicetti-Fonseca, Solera-Herrera y MoncadaJiménez, 2016; Peralta-Brenes et al., 2018).

\section{Conclusión}

Se concluye que los jóvenes universitarios de nuevo ingreso de la Universidad Veracruzana, región Veracruz, inician su etapa de educación superior con un evidente déficit en el nivel de la capacidad aeróbica. Esta conclusión es cierta para hombres y mujeres, pero especialmente para estas últimas, cuya mayoría presenta niveles deficientes de este componente de la aptitud física. Los bajos valores de capacidad aeróbica son preocupantes, debido a que esta disminuye naturalmente con la edad; sin embargo, esa reducción se puede atenuar con el debido entrenamiento físico (Kaminsky et al., 2015; Prebeg, Mihajlović y Mitić, 2012). La reducción de la capacidad aeróbica también puede ocurrir por la cantidad de horas que una persona permanece sentada estudiando durante cuatro o cinco años de estudios universitarios o, posteriormente, durante su vida laboral, lo cual aumenta el riesgo de desarrollar otras condiciones clínicas (Bouchard, Blair y Katzmarzyk, 2015).

De esta forma, es evidente la necesidad de implementar medidas de prevención y cuidado en relación con la salud cardiovascular, para evitar un posible bajo rendimiento académico, ya que mediante estudios experimentales y metaanalíticos se ha establecido una correlación positiva entre la capacidad aeróbica y los procesos cognitivos en personas de diversas edades (Hwang, Kim, Brothers, Castelli y Gonzalez-Lima, 2018; Ludyga, Gerber, Brand, Holsboer-Trachsler y Pühse, 2016). Una recomendación sencilla para mejorar 
la capacidad aeróbica es caminar, pasando progresivamente de una baja a alta intensidad, e incluso correr a baja intensidad, pues se ha demostrado en estudios epidemiológicos que con esta rutina de ejercicio se pueden reducir los factores de riesgo cardiovasculares y la mortalidad por todas las causas (Lavie et al., 2015). Por tal motivo, se sugiere continuar con el estudio en las subsiguientes generaciones, para tener un panorama de la problemática de la inactividad física de los jóvenes en el nivel medio y superior, así como para que, cuando ellos se vuelven universitarios de nuevo ingreso, tengan niveles de capacidad aeróbica que los aleje de estados que evidencian su sedentarismo.

\section{Referencias}

Alvero-Cruz, J. R., Giráldez-García, M. A. y Carnero, E. A. (2017). Reliability and accuracy of Cooper's test in male long distance runners. Revista Andaluza de Medicina del Deporte, 10(2), 60-63. DoI: https://doi.org/10.1016/j. ramd.2016.03.001

American College of Sports Medicine (ACSM). (2018). ACSM's guidelines for exercise testing and prescription (10th ed.). Philadelphia, PA: Lippincott Williams y Wilkins.

Bandyopadhyay, A. (2015). Validity of Cooper's 12-minute run test for estimation of maximum oxygen uptake in male university students. Biology of Sport, 32(1), 59-63. DOI: https://doi.org/10.5604/20831862.1127283

Bouchard, C., Blair, S. N., y Katzmarzyk, P. T. (2015). Less Sitting, More Physical Activity, or Higher Fitness? Mayo Clinic Proceedings, 90(11), 1533-1540. DoI: https://doi.org/10.1016/j.mayocp.2015.08.005

Campbell, D. T., y Stanley, J. (1963). Experimental and Quasi-Experimental Designs for Research. Boston, MA: Wadsworth Publishing.

Carpio-Rivera, E., Hernández-Elizondo, J., Salicetti-Fonseca, A., Solera-Herrera, A. y Moncada-Jiménez, J. (2016). Predictive validity of the body adiposity index in Costa Rican students. American Journal of Human Biology, 28(3), 394-397. DOI: https://doi.org/10.1002/ajhb.22800

Carpio-Rivera, E., Solera-Herrera, A., Salicetti-Fonseca, A., Hernández-Elizondo, J. y Moncada-Jiménez, J. (2016). Relación entre factores de riesgo cardiovascular y la presión arterial en reposo de estudiantes universitarios. Revista Costarricense de Salud Pública, 25(1), 30-41. Recuperado de https://www.scielo.sa.cr/scielo.php?script=sci_arttext\&pid=S1409-14292016000100047 
Carrasco-Alarcón, V., Martínez-Salazar, C., Caniuqueo-Vargas, A. y Díaz-Bustos, E. (2014). Caracterización de la Capacidad aeróbica de una muestra de estudiantes universitarios. Revista Ciencias de la Actividad Física, 15(2), 7-12. Recuperado de https://www.redalyc.org/pdf/5256/525652729001.pdf

Chastin, S. F., Mandrichenko, O., Helbostadt, J. L. y Skelton, D. A. (2014). Associations between objectively-measured sedentary behaviour and physical activity with bone mineral density in adults and older adults, the NHANES study. Bone, 64, 254-262. DoI: https://doi.org/10.1016/j.bone.2014.04.009

Cooper, K. H. (1968). A means of assessing maximal oxygen intake: correlation between field and treadmill testing. Journal of American Medical Association, 203(3), 135-138. DoI: https://doi.org/10.1001/jama.1968.03140030033008

Dana, B., Goran, P., Dusan, M. y Adela, B. (2015). Fitness index and $\mathrm{VO}_{2} \max$ of physical education students. Ovidius University Annals, Series Physical Education and Sport/Science, Movement and Health, $15(2$ S1), 246-252. Recuperado de https://www.analefefs.ro/anale-fefs/2015/i2s/pe-autori/v2/2.pdf

Das, B. (2013). Estimation of maximum oxygen uptake by evaluating Cooper 12min run test in female students of West Bengal, India. Journal of Human Sport y Exercise, 8(4), 1008-1014. DoI: https://doi.org/doi:10.4100/jhse.2013.84.11

Durán, S., Valdés, P., Godoy, A. y Herrera, T. (2014). Hábitos alimentarios y condición física en estudiantes de pedagogía en educación física. Revista Chilena de Nutrición, 41(3), 251-259. Recuperado de http://dx.doi.org/10.4067/ S0717-75182014000300004

Fernández-Ramírez, A. y Moncada-Jiménez, J. (2003). Obesidad y sobrepeso en la población estudiantil costarricense entre los 8 y 17 años. Revista Costarricense de Ciencias Médicas, 24(3,4), 95-113. Recuperado de https://www.scielo.sa.cr/ scielo.php?script=sci_arttextypid=S0253-29482003000200003

Fiuza-Luces, C., Garatachea, N., Berger, N. A. y Lucia, A. (2013). Exercise is the real polypill. Physiology (Bethesda), 28(5), 330-358. DoI: https://doi.org/10.1152/ physiol.00019.2013

Gamboa-Carmona, W. O. y Mendoza-Romero, D. (2009). Comparación de dos pruebas de resistencia aeróbica continua e intermitente en condiciones de altura intermedia en árbitros de fútbol. Lúdica Pedagógica, 1(14), 20-26. Recuperado de https://revistas.pedagogica.edu.co/index.php/LP/article/view/789

García-Laguna, D. G., García-Salamanca, G. P., Tapiero-Paipa, Y. T. y Ramos, D. M. (2012). Determinantes de los estilos de vida y su implicación en la salud de jóvenes universitarios. Revista hacia la Promoción de la Salud, 17(2), 169-185. Recuperado de http://www.scielo.org.co/pdf/hpsal/v17n2/v17n2a12.pdf 
García-Soidán, J. y Alonso-Fernández, D. (2011). Valoración de la condición física saludable en universitarios gallegos. Revista Internacional de Medicina y Ciencias de la Actividad Física y del Deporte/International Journal of Medicine and Science of Physical Activity and Sport, 11(44), 781-790. Recuperado de https://www.redalyc.org/pdf/542/54222204009.pdf

George, J. D., Fisher, A. G. y Vehrs, P. R. (2005). Laboratory experiences in exercise sciences. Burlington, MA: Jones and Bartlett Publishers, Inc.

Gómez-Figueroa, J. A., Anaya-Méndez, M., Parrazal-Cobos, J. J. y Rivera-Girón, A. R. (2015). Relación del VO2máx y la masa muscular de tudiantes de educación física, deporte y recreación. Lecturas: Educación Física y Deportes, 20(207), 1-5. Recuperado de https://dialnet.unirioja.es/servlet/articulo?codigo=5391078

Hartman, Y. A. W., Karssemeijer, E. G. A., van Diepen, L. A. M., Olde Rikkert, M. G. M. y Thijssen, D. H. J. (2018). Dementia Patients Are More Sedentary and Less Physically Active than Age- and Sex-Matched Cognitively Healthy Older Adults. Dement Geriatr Cogn Disord, 46(1-2), 81-89. DoI: https://doi. org/10.1159/000491995

Hwang, J., Kim, K., Brothers, R. M., Castelli, D. M. y Gonzalez-Lima, F. (2018). Association between aerobic fitness and cerebrovascular function with neurocognitive functions in healthy, young adults. Experimental Brain Research, 236(5), 1421-1430. DoI: https://doi.org/10.1007/s00221-018-5230-6

Kaminsky, L. A., Arena, R. y Myers, J. (2015). Reference Standards for Cardiorespiratory Fitness Measured With Cardiopulmonary Exercise Testing: Data From the Fitness Registry and the Importance of Exercise National Database. Mayo Clinic Proceedings, 90(11), 1515-1523. DoI: https://doi. org/10.1016/j.mayocp.2015.07.026

Kerr, J., Anderson, C. y Lippman, S. M. (2017). Physical activity, sedentary behaviour, diet, and cancer: an update and emerging new evidence. The Lancet Oncology, 18(8), e457-e471. DoI: https://doi.org/10.1016/s1470-2045(17)30411-4

Koury, J. C., Daleprane, J. B., Pitaluga-Filho, M. V., de Oliveira, C. F., Goncalves, M. C. y Passos, M. C. (2016). Aerobic Conditioning Might Protect Against Liver and Muscle Injury Caused by Short-Term Military Training. Journa of Strength and Conditional Research, 30(2), 454-460. DoI: https://doi. org/10.1519/jsc.0000000000001102

Lavie, C. J., Lee, D.-c., Sui, X., Arena, R., O’Keefe, J. H., Church, T. S., ... Blair, S. N. (2015). Effects of Running on Chronic Diseases and Cardiovascular and All-Cause Mortality. Mayo Clinic Proceedings, 90(11), 1541-1552. DoI: https://doi.org/10.1016/j.mayocp.2015.08.001 
Lemes, I. R., Sui, X., Fernandes, R. A., Blair, S. N., Turi-Lynch, B. C., Codogno, J. S. y Monteiro, H. L. (2019). Association of sedentary behavior and metabolic syndrome. Public Health, 167, 96-102. DoI: https://doi.org/10.1016/j. puhe.2018.11.007

Ludyga, S., Gerber, M., Brand, S., Holsboer-Trachsler, E. y Pühse, U. (2016). Acute effects of moderate aerobic exercise on specific aspects of executive function in different age and fitness groups: A meta-analysis. Psychophysiology, 53(11), 1611-1626. DOI: https://doi.org/10.1111/psyp.12736

Manso, J. M. G., Valdivielso, M. N. y Caballero, J. A. R. (1996). Bases teóricas del entrenamiento deportivo: principios y aplicaciones. Madrid: Grada Gymnos.

Mayorga-Vega, D., Bocanegra-Parrilla, R., Ornelas, M. y Viciana, J. (2016). Criterion-Related Validity of the Distance- and Time-Based Walk/Run Field Tests for Estimating Cardiorespiratory Fitness: A Systematic Review and Meta-Analysis. PLOS ONE, 11(3), e0151671. DoI: https://doi.org/10.1371/ journal.pone.0151671

Melo-Mccormick, G. y Rueda-Ochoa, O. L. (2007). Evaluación de la composición corporal y la capacidad aerobia de una muestra de estudiantes universitarios de Bucaramanga en el 2005. Revista de la Universidad Industrial de Santander. Salud, 39(2), 84-97. Recuperado de https:/www.redalyc.org/ pdf/3438/343835675002.pdf

Michell, V., Samaria, C., Junior Rudy, N., Danyela, V. y Dantas, E. (2014). Effects of a concurrent physical exercise program on aerobic power and body composition in adults. Journal of Sports Medicne and Physical Fitness, 54(4), 441-446. Recuperado de https://www.researchgate.net/publication/268220965

Miller, D. K. (1996). Measurement by the physical educator: Why and how (3rd ed.). Boston, MA: WCB McGraw-Hill.

Pareja-Galeano, H., Garatachea, N. y Lucia, A. (2015). Exercise as a Polypill for Chronic Diseases. Progresss in Molecular Biology and Translational Science, 135, 497-526. DOI: https://doi.org/10.1016/bs.pmbts.2015.07.019

Peralta-Brenes, M., Briceño-Torres, J. M., Chacón-Araya, Y., Moncada-Jiménez, J., Salazar-Villanea, M., Johnson, D. K. y Campos-Salazar, C. (2018). Prediction of Peak Aerobic Power among Costa Rican Older Adults. Journal of Clinical and Diagnostic Research, 12(11), CC01-CC04. DoI: https://doi.org/10.7860/ JCDR/2018/37095.12191

Prebeg, G., Mihajlović, N. y Mitić, D. (2012). Aerobic fitness trend of students of the Faculty of Sport and Physical Education at the University of Belgrade. 
Exercise and Quality of Life, 4(2), 41-47. Recuperado de http://scindeks-clanci.ceon.rs/data/pdf/1821-3480/2012/1821-34801202041P.pdf

Riebe, D., Franklin, B. A., Thompson, P. D., Garber, C. E., Whitfield, G. P., Magal, M. y Pescatello, L. S. (2015). Updating acsm's recommendations for exercise preparticipation health screening. Medicine and Science in Sports and Exercise, 47(8), 2473-2479. DOI: https://doi.org/10.1249/MSS.0000000000000664

Sanchis-Gomar, F., Fiuza-Luces, C. y Lucia, A. (2015). Exercise as the master polypill of the 21 st century for the prevention of cardiovascular disease. International Journal of Cardiology, 181, 360-361. DoI: https://doi. org/10.1016/j.ijcard.2014.12.048

Silfee, V., Lemon, S., Lora, V. y Rosal, M. (2017). Sedentary Behavior and Cardiovascular Disease Risk Factors among Latino Adults. Journal of Health Care for the Poor and Underserved, 28(2), 798-811. DoI: https://doi. org/10.1353/hpu.2017.0075

World Medical Association. (2014). World Medical Association Declaration of Helsinki: ethical principles for medical research involving human subjects. The Journal of the American College of Dentists, 81(3), 14. Recuperado de https:// www.ncbi.nlm.nih.gov/pmc/articles/PMC2566407/pdf/11357217.pdf 
